\title{
Variations of the solar neutrino flux
}

\author{
Yu.N.Gnedin, R.N.Ikhsanov and E.V.Miletsky \\ Central Astronomical Observatory at Pulkovo, Saint-Petersburg, Russia
}

\begin{abstract}
We present the review of temporal variations of the solar neutrino flux, including our original results of investigations of the solar neutrino flux variations in 1970-1997.
\end{abstract}

There is increasing evidence that solar neutrino flux is not constant as usually assumed for the Large-Mixing Angle (LMA) solution, but varies with different periods including well-known solar rotation and solar activity periods. Thus, the solar neutrino situation may be more complex than usually assumed, and Resonant - Spin - Flavor - Precession RSFP could be subdominant to LMA, requiring both a large transition magnetic moment and a light sterile neutrino (Coldwell \& Sturrock 2003). Here we present the additional evidences for existence of such kind variabilities with the periods longer that two year.

We used the Homestake Cl-Ar solar neutrino data (Cleveland et al. 1998) (the threshold value of $0.814 \mathrm{MeV}$ ), and GALLEX (Kisten 1999) (the threshold of $0.233 \mathrm{MeV}$ ). The Power Spectrum of Homestake data, computed by the Lomb-Scargle method, is presented in Fig. 1. One can find two peaks corresponding to the periods of 4.6 and 2.1 yrs with the confidence level $(\mathrm{CL}) \geqslant 95 \%$. One should mention the many peak situation for the neutrino flux near two year value variation that is probably due to its nonstability (Ikhsanov \& Miletsky 2003). After subtracting from the Homestake data approximately $1 / 3$ scans with the high level of noise $(>0.027$ at/day $)$ we obtain the same values of power peaks: 10, 4.6 and 2 yrs (Fig. 1 c). It means that the influence of noise is not essential.

Our conclusion is that $\sim 5$-year periodicity of the solar flux variation is most stable and therefore a real one. Here we present our conclusions about reality of this periodicity.

1. We applied the filter procedure of the Homestake data having chosen the Butterworth filter with the frequency interval of maximum transmission, corresponding to the period interval 4-14 years (Ikhsanov \& Miletsky 1999). This procedure shows that there exist six oscillations with theaverage distance between maxima 4.6 years (the GALLEX data are taken into account).

2. The Table 1 presents the mean values of the neutrino flux for all runs in a limit of $0.75 \mathrm{yr}$ from the centers of maxima and minima values of the solar neutrino flux fluctuations(I). This procedure was made also for the case when in there limits only three next runs with maximal and minimal flux magnitudes (II) were used. Table 1 shows that, firstly, all maximal values are higher as compared with two neighbor minimal. Secondly, that mean values of all maximal fluxes of solar neutrinos (No 4-10) is of 0.66 at/day. It is $45 \%$ of the solar standard model (SSM) flux values. For No 4, 8 the mean values is equal to $52 \%$ of SSM. The mean minimal value of the solar neutrino flux is only $25 \%$ of SSM. It means that modulation of the neutrino flux fluctuations can reach the level $20-25 \%$ of SSM.

3. The comparison of maxima positions with the cyclic variations of the large-scale solar magnetic field strength, on the transit of neutrinos to the Earth, implies the definite dependence. The positions of the high (No 4, 8, 12) maxima coincide with the phases of minima of 11-year cycle in the equatorial zone of large-scale magnetic field. The less values of maxima (No 6,10) are observed during the change of large-scale magnetic field 


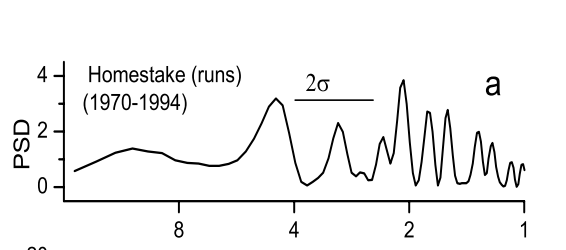

$\begin{array}{cccccc}\text { No } & \text { Date } & \begin{array}{c}\text { Max } \\ \text { at/day }\end{array} & \begin{array}{c}\text { Num. } \\ \text { of } \\ \text { runs(n) }\end{array} & \begin{array}{c}\text { Min } \\ \text { at/day }\end{array} & \begin{array}{c}\text { Num. } \\ \text { of } \\ \text { runs(n) }\end{array} \\ \text { 1 } & 1970.5 & - & & 0.27 \pm 0.20 & 4 \\ 1 \mathrm{a} & 1970.5 & - & & 0.21 \pm 0.19 & 3 \\ 2 & 1973.0 & 0.49 \pm 0.47 & 5 & - & \\ 2 \mathrm{a} & 1973.0 & 0.62 \pm 0.59 & 3 & - & \\ 3 & 1975.0 & - & & 0.44 \pm 0.31 & 6 \\ \text { 3a } & 1975.0 & - & & 0.38 \pm 0.15 & 3 \\ 4 & 1977.8 & 0.72 \pm 0.34 & 8 & - & \\ 4 \mathrm{a} & 1977.8 & 1.00 \pm 0.13 & 3 & - & \\ 5 & 1980.2 & - & & 0.17 \pm 0.19 & 8 \\ 5 \mathrm{a} & 1980.2 & - & & 0.02 \pm 0.03 & 3 \\ 6 & 1982.3 & 0.54 \pm 0.34 & 8 & - & \\ 6 \mathrm{a} & 1982.3 & 0.65 \pm 0.14 & 3 & - & \\ 7 & 1984.3 & - & & 0.48 \pm 0.34 & 9 \\ 7 \mathrm{a} & 1984.3 & - & & 0.31 \pm 0.11 & 3 \\ 8 & 1986.8 & 0.80 \pm 0.32 & 4 & - & \\ 8 \mathrm{a} & 1986.8 & 0.85 \pm 0.18 & 3 & - & \\ 9 & 1989.0 & - & & 0.49 \pm 0.30 & 9 \\ 9 \mathrm{a} & 1989.0 & - & & 0.28 \pm 0.24 & 3 \\ 10 & 1991.5 & 0.59 \pm 0.24 & 8 & - & \\ 10 \mathrm{a} & 1991.5 & 0.75 \pm 0.20 & 3 & - & \\ \text { Av. } & 4-10 & 0.66 \pm 0.31 & & 0.38 \pm 0.26 & \\ \text { Av. } & 4 \mathrm{a}-10 \mathrm{a} & 0.77 \pm 0.30 & & 0.20 \pm 0.13 & \end{array}$

Figure 1. The Power Spectrum of Homestake data

Table 1. The mean values of the neutrino flux

polarity in the equatorial zone. Atthis time, the minimal values of neutrino fluxes (No $5,10)$ correspond to the periods of high level of the solar 11-year cycle. This behavior can not be considered as accidental phenomenon.

The fluctuations of the solar neutrino flux with the periods of 10 and $\sim 5$ yrs demonstrate their tight link with the variations of solar magnetic field. The question naturally arises what is the physical reason of these variations.

The constant part of neutrino deficit is commonly accepted to explain by the theory of neutrino oscillations (MSW-effect). But the variable part of the neutrino flux deficiency can be probably connected with RSFP process that can be subdominant to LMA solution.

One can remind that the Resonant Spin-Flavour Precession (RSFP) process is based on the presence of non-zero neutrino transition magnetic moment interaction with the transverse solar magnetic field along the path of neutrino trajectory. The last data of the international project "BOREXINO" imply the new constraints of Supersymmetric (SUSY) model on the magnetic moment of neutrino: $\mu_{\nu}=5.5 \cdot 10^{-10} \mu_{B}$ for the magnetic moments of solar pp- and ${ }^{7}$ Be-neutrino.

\section{References}

Cleveland, B.T., Daily, T., Davis, R et al. 1998 Ap.J. 496, 505.

Coldwell, D.O. \& Sturrock, P.A. 2003 hep-ph/0309191.

Ikhsanov, R.N. \& Miletsky, E.V. 1999 Proceedings of the conference, Saint-Petersburg, 93

Ikhsanov, R.N. \& Miletsky, E.V. 2003 astro-ph/0312581.

Kisten, T.A. 1999 Reviews of Modern Physics 71, 1213. 